\title{
Sensitivity of the corneal-plane refractive compen- sation to change in power and axial position of an intraocular lens
}

\section{WF Harris* and RD van Gool ${ }^{\dagger}$}

\author{
Department of Optometry, University of Johannesburg, P O Box 524, Auckland Park, 2006 South \\ Africa
}

$*<$ wharris@uj.ac.za $>$

${ }^{\dagger}<$ rdvg@mweb.co.za>

Received 17 April 2009; revised version accepted 27 October 2009

\begin{abstract}
If an intraocular lens is displaced or if its power is changed what are the consequences for the refractive compensation of the eye? Gaussian optics is used to obtain explicit formulae for the sensitivity of the corneal-plane refractive compensation (also called the refraction, refractive state, etc) to change in power and axial displacement of a thin intraocular lens implanted in a simple eye. In particular, for a pseudophakic Gullstrand simplified eye with intraocular lens placed $5 \mathrm{~mm}$ behind the cornea the sensitivity to errors in the power of the intraocular lens is about -0.71 for an intraocular lens of power $20 \mathrm{D}$, that is, the refractive compensation decreases
\end{abstract}

\section{Introduction}

How important is the positioning and the power of an intraocular lens? If the lens becomes displaced or the incorrect power is used what is the consequence for the refractive compensation of that eye? An accompanying paper ${ }^{1}$ describes a general procedure for calculating the sensitivity of an optical property of an eye to change or error within the eye. The purpose of this paper is to apply the method to an eye containing an intraocular lens. More specifically the paper cal- by about 0.71 dioptres per dioptre increase in the power of the intraocular lens. More generally the sensitivity is approximately $-0.63-(0.0037 \mathrm{~m}) F_{\mathrm{I}}$ where $F_{\mathrm{I}}$ is the power of the intraocular lens. Also for Gullstrand's simplified eye the sensitivity of refractive compensation to axial displacement of the intraocular lens is approximately linear in $F_{\mathrm{I}}$, about (64D) $F_{\mathrm{I}}$, in fact. That is, for each dioptre of the power of the intraocular lens the refractive compensation increases by about 0.064 dioptres per millimetre of axial displacement towards the retina.

Key words: intraocular lens, sensitivity, axial displacement, Gaussian optics, refraction, refractive compensation, Gullstrand's simplified eye.

*PhD FRSSAf

†DPhil

culates the sensitivity of the corneal-plane refractive compensation of the eye to change in power and axial position of the intraocular lens. In order to display the ideas, minimally obscured by mathematics, use is made of the simplest reasonable optics (Gaussian optics) and the simplest pseudophakic eye (a single refracting surface for the cornea and a thin intraocular lens). For detail on the linear optics of the pseudophakic eye, not necessarily thin, and on the effect of axial position of the intraocular lens the reader is referred elsewhere ${ }^{2,3}$. 


\section{Pseudophakic simplified eye}

Consider a pseudophakic simplified eye. The eye can be regarded as consisting of two systems, the anterior part $\mathrm{S}_{\mathrm{A}}$ and the posterior part $\mathrm{S}_{\mathrm{P}}$ (Figure 1). The anterior part has entrance plane $\mathrm{T}_{0}$ immediately in front of the cornea $\mathrm{K}$ and exit plane $\mathrm{T}_{\mathrm{I}}$ immediately in front of the intraocular lens I. It consists of a thin system (the cornea) of power $F_{\mathrm{K}}$ followed by a homogeneous gap of reduced width $\zeta_{\mathrm{A}}$. The posterior part has entrance plane $T_{I}$ and exit plane $T$ immediately in front of the retina $\mathrm{R}$. Its structure is optically similar: it also consists of a thin system (an intraocular lens I) of power $F_{\text {I }}$ and a homogeneous gap of reduced width $\zeta_{\mathrm{P}}$. The index of refraction is $n$ within the eye and 1 in front of the eye.

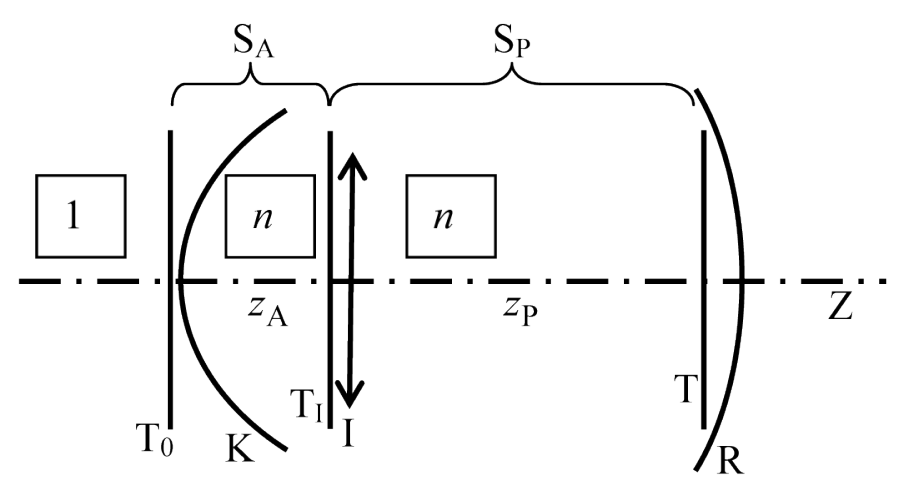

Figure 1 A simplified eye with cornea $\mathrm{K}$ of power $F_{\mathrm{K}}$ and an implanted thin intraocular lens I of power $F_{\mathrm{I}}$. The distance between the cornea and the intraocular lens is $z_{\mathrm{A}}$ and between $\mathrm{I}$ and the retina $\mathrm{R}$ is $z_{\mathrm{P}} . \mathrm{S}_{\mathrm{A}}$ is the anterior part and $\mathrm{S}_{\mathrm{P}}$ the posterior part of the eye.

Each part of the eye has a transference of the form given by Equation 19 of the accompanying paper ${ }^{1}$; for the anterior part

$$
\mathbf{S}_{\mathrm{A}}=\left(\begin{array}{cc}
1-\zeta_{\mathrm{A}} F_{\mathrm{K}} & \zeta_{\mathrm{A}} \\
-F_{\mathrm{K}} & 1
\end{array}\right)
$$

and for the posterior part

$$
\mathbf{S}_{\mathrm{P}}=\left(\begin{array}{cc}
1-\zeta_{\mathrm{P}} F_{\mathrm{I}} & \zeta_{\mathrm{P}} \\
-F_{\mathrm{I}} & 1
\end{array}\right)
$$

It follows from Equation 11 of that paper that the transference of the pseudophakic eye is
$\mathbf{S}=\left(\begin{array}{cc}1-\zeta_{\mathrm{P}} F_{\mathrm{I}} & \zeta_{\mathrm{P}} \\ -F_{\mathrm{I}} & 1\end{array}\right)\left(\begin{array}{cc}1-\zeta_{\mathrm{A}} F_{\mathrm{K}} & \zeta_{\mathrm{A}} \\ -F_{\mathrm{K}} & 1\end{array}\right)$

which multiplies out to

$\mathbf{S}=\left(\begin{array}{cc}\left(1-\zeta_{\mathrm{P}} F_{\mathrm{I}}\right)\left(1-\zeta_{\mathrm{A}} F_{\mathrm{K}}\right)-\zeta_{\mathrm{P}} F_{\mathrm{K}} & \left(1-\zeta_{\mathrm{P}} F_{\mathrm{I}}\right) \zeta_{\mathrm{A}}+\zeta_{\mathrm{P}} \\ \bullet & \bullet\end{array}\right)$

The bottom row of the matrix is not needed below and is omitted.

\section{Sensitivity of the refractive compensation to change in power of the intraocular lens}

Applying Equation 4 above to Equation 16 of the previous paper ${ }^{1}$ one obtains the corneal-plane refractive compensation

$$
F_{0}=\frac{1-\zeta_{\mathrm{P}} F_{\mathrm{I}}}{\zeta_{\mathrm{A}}+\zeta_{\mathrm{P}}-\zeta_{\mathrm{A}} \zeta_{\mathrm{P}} F_{\mathrm{I}}}-F_{\mathrm{K}}
$$

(The denominator, with form reminiscent of the form of what is commonly called the equivalent power of a thick lens, might be regarded as an equivalent or effective reduced length of the eye.) Equation 5 defines $F_{0}$ as a function $F_{0}^{F_{\mathrm{K}} \zeta_{\mathrm{A}} F_{\mathrm{I}} \zeta_{\mathrm{P}}}$ of the four independent variables $F_{\mathrm{K}}, \zeta_{\mathrm{A}}, F_{\mathrm{I}}$ and $\zeta_{\mathrm{P}}$. It also implies four derivatives, that is, four sensitivities. One of them is obvious: the sensitivity of the compensation to change in corneal power, that is, $F_{0}^{F_{\mathrm{K}}^{\prime} \zeta_{\mathrm{A}} F_{\mathrm{I}} \zeta_{\mathrm{P}}}$, is -1 , in agreement with what we would expect.

We are interested in the sensitivity of the cornealplane refractive compensation to change in power of the intraocular lens, that is $F_{0}^{F_{\mathrm{K}} \zeta_{\mathrm{A}} F_{\mathrm{I}}^{\prime} \zeta_{\mathrm{P}}}$. Differentiation of Equation 5 results in

$$
F_{0}^{F_{\mathrm{K}} \zeta_{\mathrm{A}} F_{\mathrm{I}}^{\prime} \zeta_{\mathrm{P}}}=-1 /\left(1+\zeta_{\mathrm{A}} / \zeta_{\mathrm{P}}-\zeta_{\mathrm{A}} F_{\mathrm{I}}\right)^{2}
$$

This shows in particular that the sensitivity is independent of the corneal power $F_{\mathrm{K}}$.

\section{Sensitivity of the refractive compensation to axial displacement of the intraocular lens}

Suppose instead one is interested in the sensitivity of the corneal-plane refractive compensation to 
change in axial position of the intraocular lens. We need to work in terms of distances instead of reduced distances. Equation 5 can be expressed as

$$
F_{0}=\frac{n-z_{\mathrm{P}} F_{\mathrm{I}}}{z_{\mathrm{A}}+z_{\mathrm{P}}-z_{\mathrm{A}} z_{\mathrm{P}} F_{\mathrm{I}} / n}-F_{\mathrm{K}} .
$$

$z_{\mathrm{A}}$ and $z_{\mathrm{P}}$ are the widths of the anterior and posterior parts of the eye respectively.

Axial displacement of the lens changes both $z_{\mathrm{A}}$ and $z_{\mathrm{P}}$. One needs to find an independent variable that can be changed while all others remain fixed. An obvious procedure is to define $\mathrm{z}$ the length of the eye:

$z=z_{\mathrm{A}}+z_{\mathrm{P}}$

Substituting $z-z_{\mathrm{A}}$ for $z_{\mathrm{P}}$ in Equation 7 and rearranging we obtain

$$
F_{0}=\frac{n-\left(z-z_{\mathrm{A}}\right) F_{\mathrm{I}}}{z-\left(z-z_{\mathrm{A}}\right) z_{\mathrm{A}} F_{\mathrm{I}} / n}-F_{\mathrm{K}} \text {. }
$$

This represents the function $F_{0}^{F_{\mathrm{K}} z_{\mathrm{A}} z n F_{\mathrm{I}}}$ of the five independent variables $z_{\mathrm{A}}, F_{\mathrm{K}}, F_{\mathrm{I}}, z$ and $n$ and each of the five derivatives represent sensitivities.

In order to find the sensitivity of the corneal-plane refractive compensation to change in distance $z_{\mathrm{A}}$ with $F_{\mathrm{K}}, F_{\mathrm{I}}, z$ and $n$ constant we need to differentiate with respect to $z_{\mathrm{A}}$. The result turns out to be

$$
F_{0}^{F_{\mathrm{K}} z_{\mathrm{A}}^{\prime} z n F_{\mathrm{I}}}=\frac{n\left(z-z_{\mathrm{A}}\right)\left(2 n-\left(z-z_{\mathrm{A}}\right) F_{\mathrm{I}}\right) F_{\mathrm{I}}}{\left(n z-z z_{\mathrm{A}} F_{\mathrm{I}}+z_{\mathrm{A}}^{2} F_{\mathrm{I}}\right)^{2}}
$$

a sensitivity which, as expected, is also independent of the corneal power.

\section{Application to a pseudophakic simplified eye}

Consider Gullstrand's simplified eye from which the lens has been removed. The index of the aqueous and vitreous humors is $n=1.336$, the length of the eye is $z=24 \mathrm{~mm}$ and the power of the cornea is $F_{\mathrm{K}}=$ 48.03D. ${ }^{4}$
Suppose a thin intraocular lens is implanted at a distance of $z_{\mathrm{A}}=5 \mathrm{~mm}$ behind the cornea. Hence $z_{\mathrm{P}}=$ $19 \mathrm{~mm}$ and the reduced widths are $\zeta_{\mathrm{A}}=3.74 \mathrm{~mm}$ and $\zeta_{\mathrm{P}}=14.22 \mathrm{~mm}$. The corneal-plane refractive compensation is given by Equations 5, 7 or 9; for an implant of power $F_{\mathrm{I}}=20 \mathrm{D}$ the corneal-plane refractive compensation turns out to be $-0.74 \mathrm{D}$.

Equation 6 results in

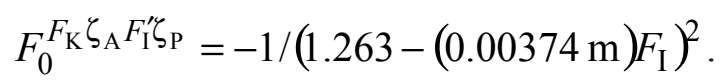

Because the coefficient of $F_{\mathrm{I}}$ in the denominator is in metres $F_{\mathrm{I}}$ is necessarily in dioptres. For $F_{\mathrm{I}}=20 \mathrm{D}$ this gives a sensitivity of about -0.71 . One can think of this as $-0.71 \mathrm{D} / \mathrm{D}$. Thus for $1 \mathrm{D}$ increase in power of the intraocular lens the corneal-plane refractive compensation will decrease by about $0.71 \mathrm{D}$, thus changing the compensation to an estimated value of about

-1.45 D. (This agrees with the true compensation calculated via Equation 5 for $F_{\mathrm{I}}=21 \mathrm{D}$.) A decrease of $1 \mathrm{D}$ results very nearly in emmetropia. For $F_{\mathrm{I}}=$ $10 \mathrm{D}$ the sensitivity is -0.67 and for $F_{\mathrm{I}}=30 \mathrm{D}$ it is -0.75 . The dependence of sensitivity on the power of the intraocular lens is roughly affine, the binomial expansion being

$$
F_{0}^{F_{\mathrm{K}} \zeta_{\mathrm{A}} F_{\mathrm{I}}^{\prime} \zeta_{\mathrm{P}}} \approx-0.63-(0.0037 \mathrm{~m}) F_{\mathrm{I}}
$$

From Equation 10 we obtain the sensitivity of the refractive compensation to change in axial position. For $F_{\mathrm{I}}=20 \mathrm{D}$ the sensitivity is about $1280 \mathrm{D}^{2}$ or about $1.28 \mathrm{D} / \mathrm{mm}$. An axial shift towards the retina of $1 \mathrm{~mm}$ will increase the refractive compensation by about $1.28 \mathrm{D}$ to an estimated compensation of about D. The actual refractive compensation, calculated via Equation 5, is $0.52 \mathrm{D}$. Hence the error in the estimate is $0.02 \mathrm{D}$. For $F_{\mathrm{I}}=10 \mathrm{D}$ the sensitivity is about 650 $\mathrm{D}^{2}$ or $0.65 \mathrm{D} / \mathrm{mm}$ and for $F_{\mathrm{I}}=30 \mathrm{D}$ it is about 1880 $\mathrm{D}^{2}$. In fact the sensitivity is approximately linear in $F_{\mathrm{I}}$ :

$F_{0}^{F_{\mathrm{K}} z_{\mathrm{A}}^{\prime} z n F_{\mathrm{I}}} \approx(64 \mathrm{D}) F_{\mathrm{I}}$.

Thus for every dioptre of power of the intraocular lens the corneal-plane refractive compensation increases 
by about $0.064 \mathrm{D}$ per millimetre of axial displacement towards the retina.

\section{Concluding remarks}

Explicit formulae have been derived here, using Gaussian optics, for a pseudophakic eye derived from Gullstrand's simplified eye. The formulae give the sensitivity of the corneal-plane refractive compensation to error or change in axial position (Equation 10) and power (Equation 5) of an intraocular lens. Sensitivity to change or error in power is a relatively-weak and approximately-affine function of the power of the intraocular lens (Equation 12) whereas sensitivity to axial displacement is approximately linear in the power (Equation 13).

\section{Acknowledgements}

WF Harris thanks the National Research Foundation for support.

\section{References}

1. Harris WF. General approach to the sensitivity of the optics of an eye to change in elementary parameters with application to the Gaussian optics of a reduced eye. S Afr Optom 68 166-174.

2. MacKenzie GE. Linear Optics of the Pseudophakic Eye. DPhil dissertation, Rand Afrikaans University, Johannesburg 2004

3. MacKenzie GE, Harris WF. Solving for intraocular lens position from stigmatic and astigmatic data. S Afr Optom 200362 135-138.

4. Gullstrand A. IV. Der Mechanismus der Akkommodation. In: van Helmholtz H, Handbuch der Physiologischen Optik, 3rd ed, Leopold Voss, Hamburg, 1909, pp 327-353. 